\title{
A hybrid solar panel maximum power point search method that uses light and temperature sensors in real shading conditions
}

\author{
J. Mroczka, M. Ostrowski \\ Chair of Electronic and Photonic Metrology \\ Wroclaw University of Technology \\ B. Prusa 53/55, 50-317 Wroclaw, Poland \\ Phone/Fax number: +48 713206232 \\ E-mail: janusz.mroczka@pwr.edu.pl, mariusz.ostrowski@pwr.edu.pl
}

\begin{abstract}
Solar panels are common renewable energy sources. They generate electrical energy directly from the solar energy but have low efficiency and non-linear characteristics. To decrease the power loss solar panels are connected to special devices called maximum power point (MPP) trackers. When the surface of the solar panel is illuminated irregularly in the powervoltage curve can be observe the appearance of the local maximum power points that are different from the global maximum power point. In the partial shading condition the regular trackers can track the local maximum power point. The presented method use the light and temperature measurement to determine the global maximum power point. The simulation results prove correctness of proposed method in real shading conditions. The proposed algorithm has high tracking speed and does not depend of used solar panels, and by that can be used in any solar system.
\end{abstract}

\section{Key words}

Maximum power point tracking algorithm, illumination measurement, temperature measurement, real shading conditions.

\section{Introduction}

The output power of the solar panel is dependent on illumination and temperature, as presented in Fig. 1. When the temperature increases the maximum power point voltage value decreases and is equal approximately to $80 \%$ of open circuit voltage [1]. The open circuit voltage can be estimated on the bases of temperature value and the temperature coefficient given by the manufacturer.

When the illumination increase the maximum power point current increasing. The value of the maximum power point current is approximately equal to $90 \%$ of the short circuit current [1], and its value can be estimated by using the illumination value from sensor.

To increase the output power from the solar system solar cells are connected in series creating the solar module. The solar modules are connected in more complex structures creating grid of series or parallel connected modules. To avoid negative effect of partial shading the solar modules or solar cells are connected parallel with bypass diodes.
This connection can make appearance of the local maxima on power-voltage curve.

The regular maximum power point search algorithm cannot properly search the global maximum power point. The modified algorithms search entire power-voltage curve by constant or adaptive step to determinate the global maximum power point. Another methods use measurement of the open circuit voltage or the short circuit current and its division to determine the maximum power point. This can cause the power loss.
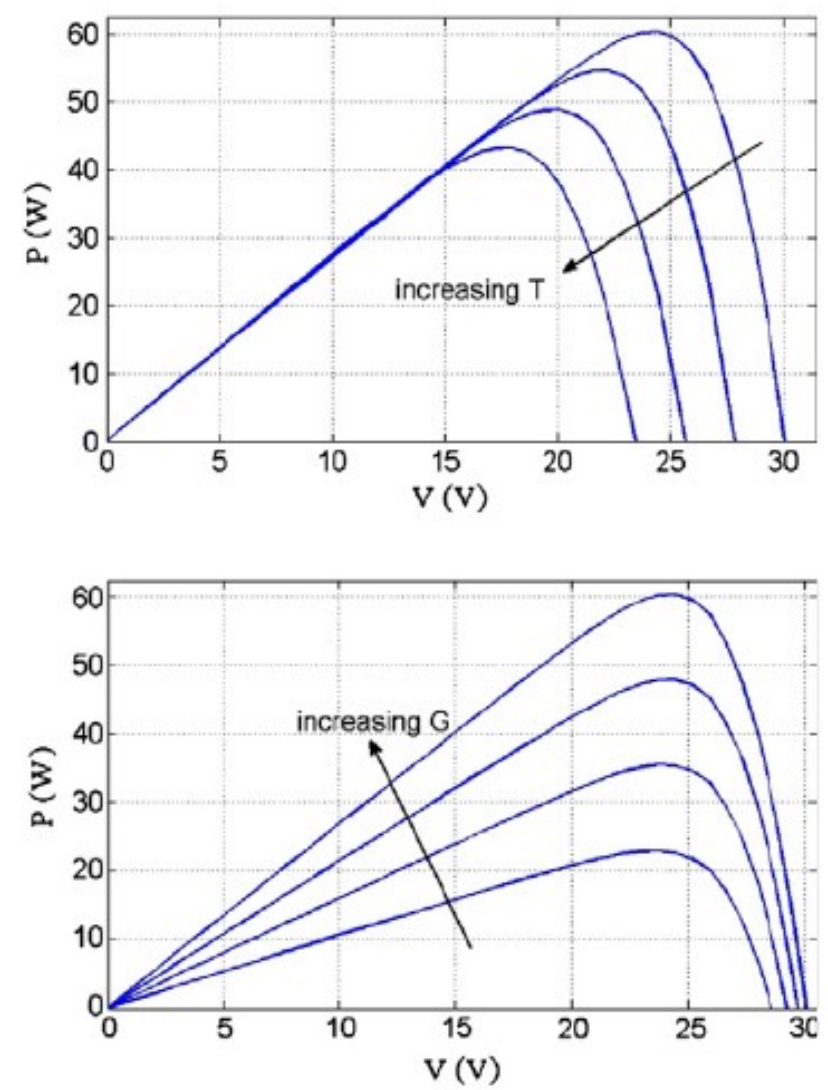

Fig. 1. The relationship between the temperature and illumination and the power in the maximum power point. 


\section{Proposed method}

There are few maximum power point search method that can track global maximum power point in literature [10-15]. Many of them searching approximate position of global maximum power point on the bases of the open circuit voltage or temperature and on this bases run traditional perturb and observe method. The proposed method consists of two sub-algorithms: the algorithm that uses a light sensor, current method, in equal illumination conditions and the algorithm that uses temperature sensors, temperature method, in partial shading conditions. Both of them are adaptive and use the correlation function to determine the maximum power point. Both methods was described in our previous work [4-5]. The block diagram of the proposed method is presented on Fig. 2.

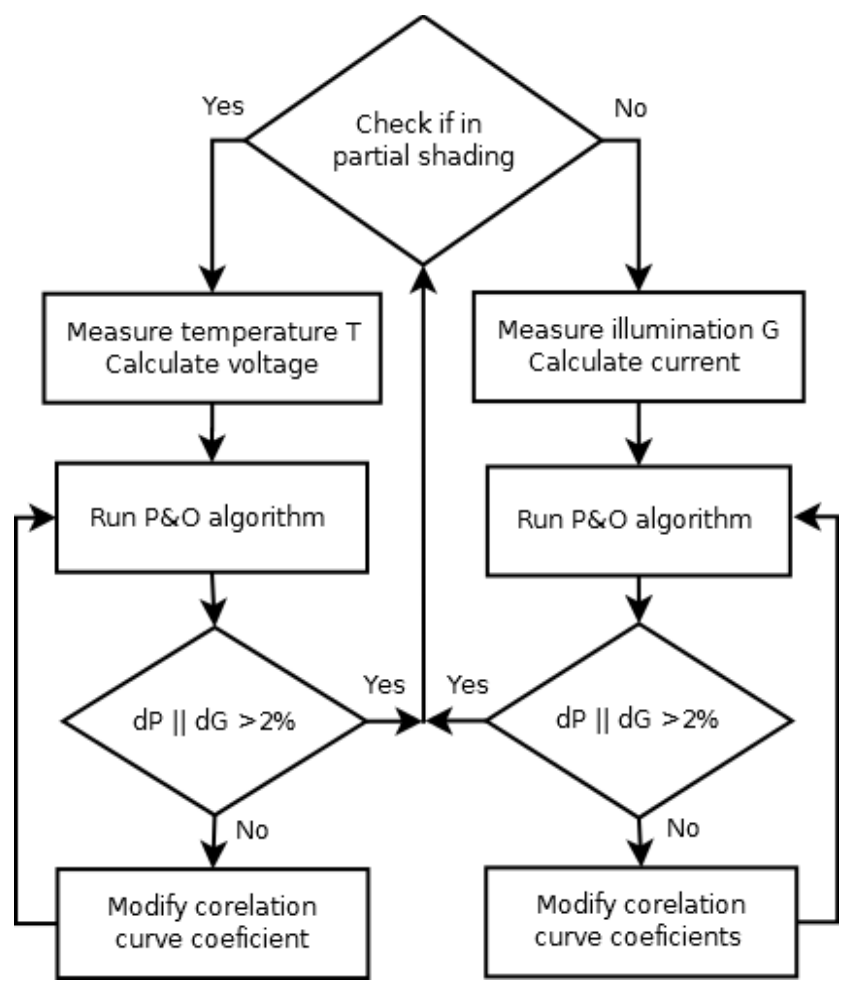

Fig. 2. The block diagram of the proposed method.

The correlation function is implemented by the table of the line function coefficients. Each sub-algorithm has its own table of coefficients. The value of the correlation function coefficient is modified on base of the error between the calculated and the real maximum power point. Each of the sub-algorithm is presented below.

Each of sub-algorithms is running until power, temperature or illumination change is lower than $2 \%$. When the step of the perturb and observe (P\&O) method is equal to $1 \%$ of the initial step the error value between calculated and measured current is used to modify the correlation function coefficient.

On the beginning when the correlation coefficient are not calculated, the algorithm seek whole power-voltage curve to find the maximum power point. On its base the algorithm modify the correlation coefficient tables. After that algorithm interpolate missing values using the line function.

\section{A. Algorithm that uses illumination sensor}

In regular illumination conditions the maximum power point position is dependent of illumination and temperature. This method is fast and independent of used illumination sensor and solar panel [4]. The algorithm uses the value of the illumination sensor and on this base calculates the current in maximum power point, Eq. (1),

$$
I_{M P P}=a_{I} G+b_{I} \text {, }
$$

where $I_{M P P}$ is maximum power point current, $a_{I}$ and $b_{I}$ are the line function coefficients and $G$ is the measured illumination value. On the base of the calculated current value the perturb and observe algorithm[8,9] with adaptive step size is running until there is no change in power or illumination.

\section{B. Algorithm that uses temperature sensor}

In partial shading conditions algorithm calculate maximum power point voltage on the bases of the value of the temperature sensors[10]. The temperature sensors and parameters given by manufacturer to calculate each of the local maximum power points were used in the previous work [5], Eq. (2),

$$
V_{L M P P, m}=\sum_{n=1}^{m} 0.8 k_{T} T_{n},
$$

where $V_{L M P P, m}$ is the $m$-th local maximum power point voltage, $k_{T}$ is the temperature coefficient given by the manufacturer, $T_{n}$ is the value of $n$-th temperature sensor. In the presented method the value of the $m$-th local maximum power point voltage value is calculated by using $m$-th temperature sensor value and correlation function coefficient, Eq. (3),

$$
V_{L M P P, m}=\sum_{n=1}^{m} a_{T} T_{n}+b_{T},
$$

where $a_{T}$ and $b_{T}$ are the coefficients of correlation function.

\section{Simulation results}

Simulation were performed for solar panel consists of six series connected MSX-60PV solar modules with bypass diodes, each for one module. The MSX-60PV parameters are shown in table I.

Table I. - Parameters of MSX-60PV module

\begin{tabular}{|l|l|l|}
\hline \multicolumn{1}{|c|}{ Parameter } & Variable & \multicolumn{1}{c|}{ Value } \\
\hline Maximum power & PMPP & $60 \mathrm{~W}$ \\
\hline Voltage at MPP & VMPP & $17.1 \mathrm{~V}$ \\
\hline Current at MPP & IMPP & $3.5 \mathrm{~A}$ \\
\hline Open circuit voltage & VOC & $21.1 \mathrm{~V}$ \\
\hline Short circuit current & ISC & $3.8 \mathrm{~A}$ \\
\hline $\begin{array}{l}\text { Temperature coefficient of } \\
\text { VOC }\end{array}$ & $\mathrm{KV}$ & $-80 \pm 10 \mathrm{mV} / \mathrm{K}$ \\
\hline
\end{tabular}




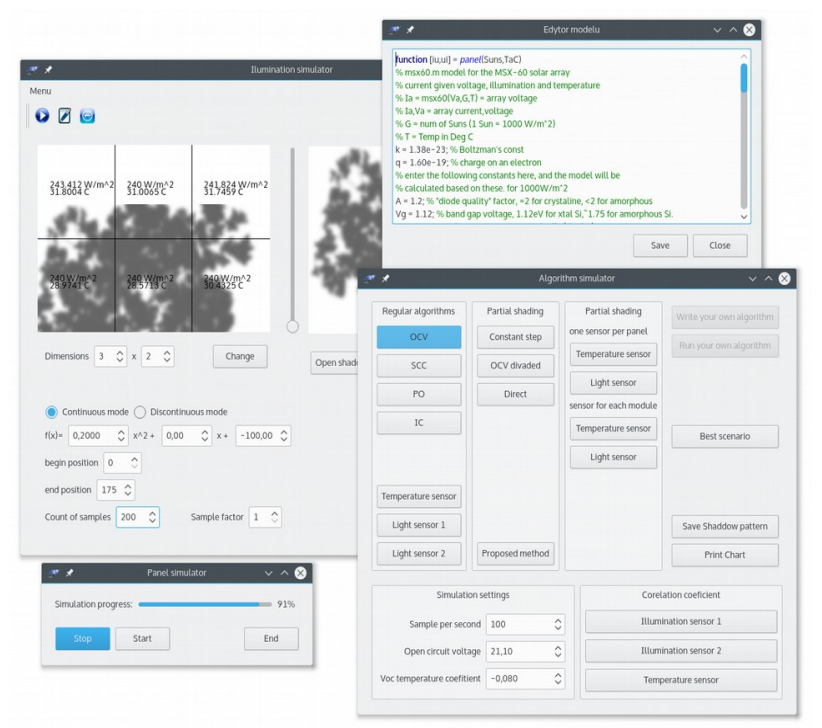

Fig. 3. The simulation workbench.

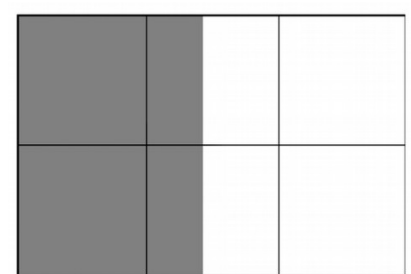

a)

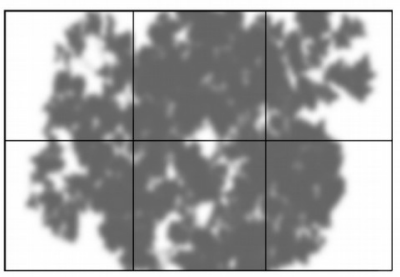

c)

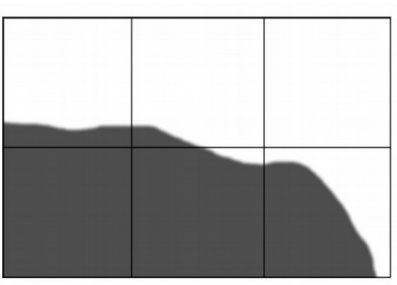

b)

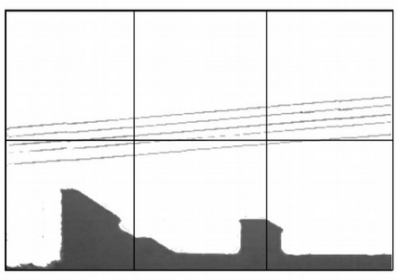

d)
Fig. 4. The shadow patterns: a) block shadow, b) shadow caused by cloud, c) shadow caused by tree, d) shadow caused by building

\section{A. Algorithm simulation workbench}

The algorithm simulation workbench was created by authors and was presented during the ICREPQ conference in Cordoba in 2104 [16]. The software allow to apply any shadow pattern, calculate the illumination and temperature for each solar module and on its bases calculate the output power, simulate any of the maximum power point search algorithm, test them and compare with each other in the same solar and temperature conditions. The simulation workbench is shown in Fig. 3. It uses any of solar panel model written in Matlab/Octave script file. In simulation the one diode model written by González-Longatt was used $[7,8]$.

\section{B. Shadow patterns}

The simulation were proved for the real shadow patterns, see Fig. 4 for different ambient temperature and illuminations. The patterns was made by digital camera on the white material that simulate the surface of solar panel.

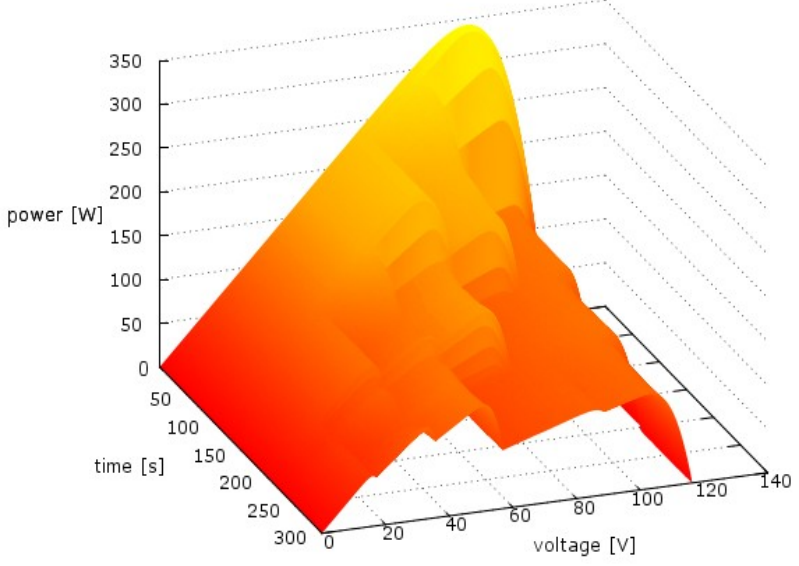

Fig. 5. The output power-voltage characteistic for shadow pattern cosed by tree.

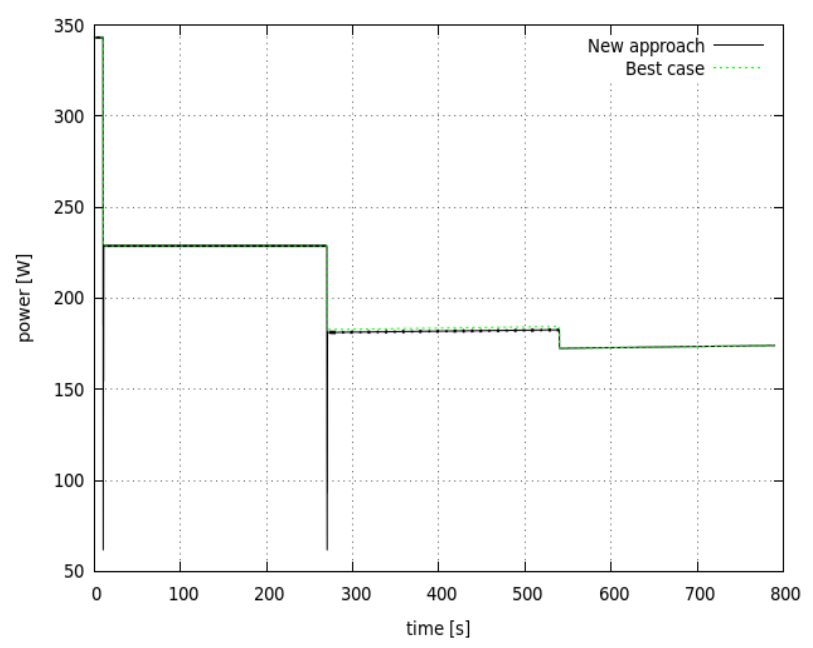

Fig. 6. The output power-time characteristic for a block shadow pattern.

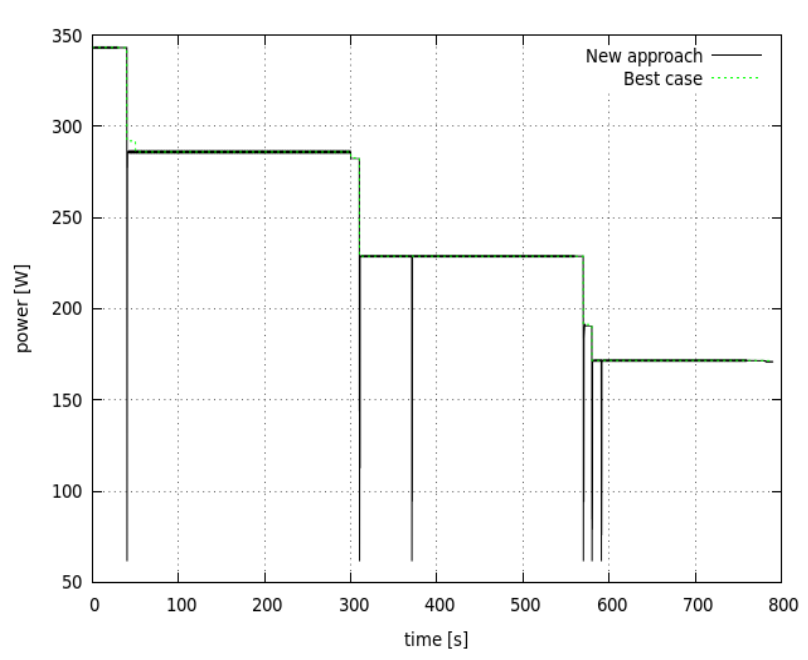

Fig. 7. The output power-time characteristic for a shadow pattern caused by cloud.

Those are common shades - block shadow can be found in highly urbanized places, clouds, tree and roof of the house. The simulated output power-voltage characteristic 


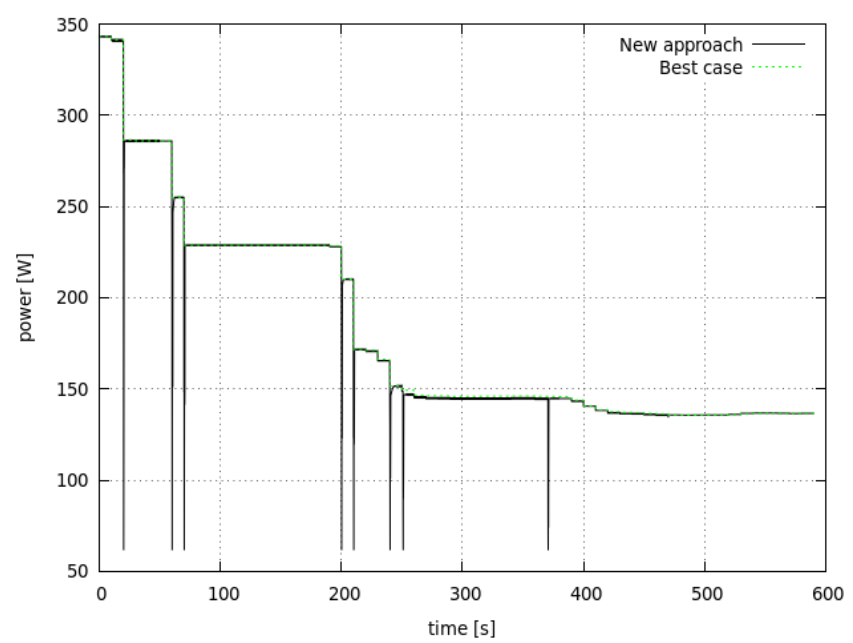

Fig. 8. The output power-time characteristic for a shadow pattern cosed by tree.

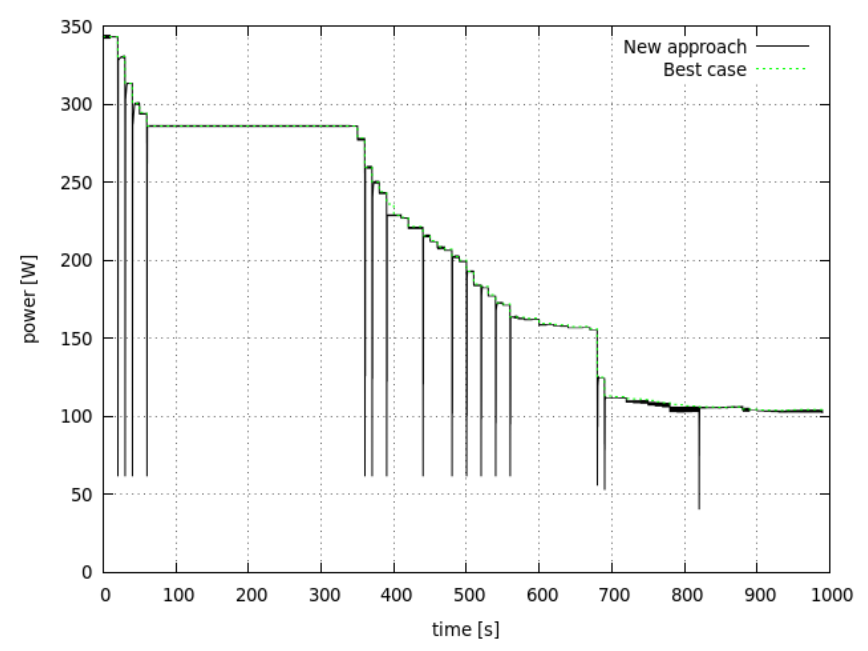

Fig. 9. The output power-time characteristic for a shadow pattern cosed by building.

for the shadow caused by tree is presented on the Fig. 5. It can bee seen the appearance of local maxima.

\section{Simulation results}

On the beginning, when the coefficient function table is empty the algorithm has speed equal to regular perturb and observe method. The initial step is equal to the open circuit voltage divided by numbers of bypass diodes. Algorithm measure power in points corresponding to sum of initial step size. After that the initial step is divided by 2 and algorithm is running from the point with maximum power. The algorithm calculates the correlation coefficient. In the simulation the correlation coefficient table is calculated.

In the Fig. 6 can be seen the output power of photovoltaic panel for block shadow pattern. The proposed method on the beginning use the solar sensor (the illumination is equal for each module) to calculate the maximum power point current. The seeking error does not exceed $0.5 \%$. The perturb and observe method with variable step size quickly reach the maximum power point. When output power change algorithm checking if there are equal illumination for whole panel. In this case the illumination is not equal so the proposed algorithm run the temperature subalgorithm. For each temperature sensor the local maximum power point voltage value is calculated and the output power is checked. For the voltage witch have the largest power the perturb and observe method is running. The error between real and calculated maximum power point do does not exceed $1 \%$. The number of testing points is equal to number of bypass diodes (each temperature sensor for each bypass diode). For each case the perturb and observe method is running until the power or temperature or illumination is changing. The output power is almost $99 \%$ of possible power.

The same appearance for proposed method can be seen on the Fig. 7. In the beginning the maximum power point current is calculated on the bases of value of illumination. When the shadow becomes on the surface of the panel the output power is decreasing and algorithm checking if the power drop is caused by the shadow. If so, the second sub-algorithm is running, see Fig. 7.

The proposed method is correct even for complex shadow pattern. In the Fig. 8 can be seen the output power caused by shadow of the tree. The proposed method always find the real maximum power point. The output power is almost $98 \%$ of possible power. Even on the shadow caused by the building, see Figs. 5 and 9, algorithm find the real maximum power point.

Due to using solar sensor proposed method is very fast in uniform illumination condition. The seeking error does not reach $1 \%$ of real maximum power point current in every simulation pattern. Due to using adaptive correlation coefficient the proposed method do not depended on used illumination sensor and solar panel schema. By using temperature sensors proposed method is very fast in partial shading condition. Even there are some inert ion on solar sensor behaviour the seeking error do not reach $2 \%$ of maximum power point voltage value. Due using the perturb and observe method with adaptive step size any seeking errors are very fast corrected.

\section{Conclusion}

The simulation results prove correctness of the proposed method in real shading conditions. The simulation results show, that proposed method is fast and accurate even for very complex shadow patterns. Both of used algorithms are independent of used solar panels or sensors, and can be used in any solar system. By using two sub-algorithm the tracking speed increase in comparison to algorithms presented in our previous works.

The method that uses solar sensor is very fast and accurate for the uniform insolation conditions, and this condition is the most common condition, but using many solar sensor is uneconomical. The method that uses temperature sensors is fast in partial shading condition and by using correlation function can be used in every 
solar system. By using both methods proposed algorithm is very fast and flexible.

\section{Acknowledgement}

We would like to acknowledge support of this work by Polish Ministry of Science and Higher Education (0402/0015/15 - B50038).

\section{References}

[1] V. Salas, E. Olias, A. Barrado, and A. Lazaro, "Review of the Maximum Power Point Tracking Algorithms for StandAlone Photovoltaic Systems," Solar Energy Materials and Solar Cells 90, 1555 (2006)

[2] G. Petrone, G. Spagnuolo, R. Teodorescu, M. Veerachary, and M. Vitelli, "Reliability Issues in Photovoltaic Power Processing Systems," IEEE Transactions on Industrial Electronics 55, 2569 (2008)

[3] A. N. A. Ali, M. H. Saied, M. Z. Mostafa and T. M. AbdelMoneim, "A Survey of Maximum PPT Techniques of PV Systems,” Energytech 2012 IEEE 1, 17 (2012)

[4] M. Ostrowski, "Photovoltaic maximum power point search method using a light sensor", Integrated Optics: Physics and Simulations II / Pavel Cheben, Jiří Čtyroký, Iñigo MolinaFernández (eds.). Bellingham, Wash. : SPIE, cop. 2015. art. 95160D, s. 1-8.

[5] J. Mroczka and M. Ostrowski, "A hybrid maximum power point search method using temperature measurements in partial shading conditions.”, Metrology and Measurement Systems. 2014, vol. 21, nr 4, s. 733-740.

[5] M. H. Moradi, and A.R. Reisi, "A Hybrid Maximum Power Point Tracking Method for Photovoltaic Systems”, Solar Energy 85(11), 2965-2976 (2011)

[6] F. M. González-Longatt, "Model of Photovoltaic Module in MatlabTM", II CIBELEC 2006, 2006, pp. 1-5.

[7] Sera D., Teodorescu R., Rodriguez P., PV panel model based on datasheet values, Industrial Electronics, 2007. ISIE 2007. IEEE International Symposium on, 4-7 June 2007, 2392 2396.

[8] Salas V. , Olias E., Barrado A, Lazaro A., "Review of the maximum power point tracking algorithms for stand-alone photovoltaic systems”, Sol. Energy Mater. Sol. Cells, vol. 90, p.1555, 2006

[9] Ali, A. N. A., Saied, M. H., Mostafa, M. Z., Abdel-Moneim, T. M.. "A Survey of Maximum PPT Techniques of PV Systems”. Energytech 2012 IEEE, 1,17. (2012)

[10] Coelho, R. F., Concer, F. M., Martins, D. C. “A MPPT Approach Based on Temperature Measurements Applied in PV Systems. Proc. IEEE ICSET, 1-6.(2010)

[11] Patel, H., Agarwal, V. "Maximum Power Point Tracking Scheme for PV Systems Operating Under Partially Shaded Conditions”. IEEE Trans. Ind. Electron., 55, 1689-1698. (2008)

[12] Park, M., Yu, I. A. "Study on the Optimal Voltage for MPPT Obtained by Surface Temperature of Solar Cell”. Proc. IECON, 30, 2040-2045. (2004)

[13] Nguyen, T. L., Low, K. S. A Global Maximum Power Point Tracking Scheme Employing DIRECT Search Algorithm for Photovoltaic Systems. Ieee Transactions On Industrial Electronics, 57(10). (2010)

[14] Moradi, M. H., Reisi, A. R. "A Hybrid Maximum Power Point Tracking Method for Photovoltaic Systems”. Solar Energy, 85(11), 2965-2976. (2011)

[15] Koutroulis, E., Blaabjerg, F. “A New Technique for Tracking the Global Maximum Power Point of PV Arrays Operating Under Partial-Shading Conditions”. IEEE J. Photovoltaics, 2(2), 184-190.( 2012)
[16] Mroczka J., Ostrowski M. "Photovoltaic array simulation technique for non-uniform insolation conditions", Renewable Energy \& Power Quality Journal nr 12, s. 1-5 (2014)

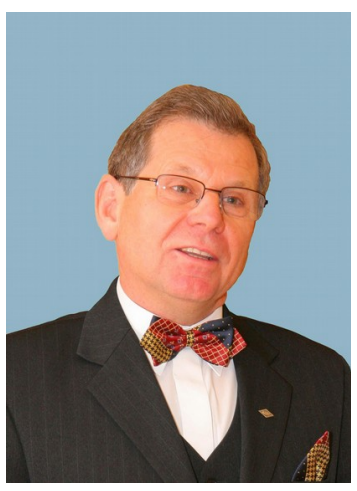

Janusz Mroczka is a professor of electronic and photonic metrology at Wroclaw University of Technology. He was born in Poland in 1952. In 1991, he received the higher doctor's degree (habilitation) for a thesis in metrological problems of scattered light application on particle size distribution investigation in dispersed solutions, from the same university. Since 1998, he has been the head of the Chair of Electronic and Photonic Metrology at Wroclaw University of Technology.

His research interests are concentrated on physical and mathematical modeling in complex measurements, inverse problem, intelligent measuring devices, scattering radiation spectral and polarization analysis, and multisensor data fusion and time-frequency representations in data processing.

Professor Mroczka is a member of SPIE, a member of the Polish Physical Society, and President of the Committee for Metrology and Instrumentation at the Polish Academy of Science. Since 2010, he has been a Corresponding Member of the Polish Academy of Science

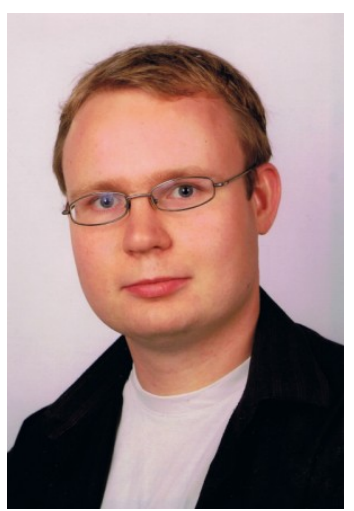

Mariusz Ostrowski is a $\mathrm{PhD}$ student of electronics at Wroclaw University of Technology. Since 2010, he has worked on modeling and optimization process of obtaining energy from photovoltaic sources.

His main interests are electronics of renewable energy and computer science. 